\title{
Non-uniform Mutation Rates for Problems with Unknown Solution Lengths
}

\author{
Stephan Cathabard \\ CIRRELT \\ University of Montreal \\ Montreal, Canada \\ cathabas@iro.umontreal.ca
}

\author{
Per Kristian Lehre \\ DTU Informatics \\ 2800 Lyngby, Denmark \\ pkle@imm.dtu.dk
}

\author{
Xin Yao \\ University of Birmingham \\ Birmingham B15 2TT, UK \\ x.yao@cs.bham.ac.uk
}

\begin{abstract}
Many practical optimisation problems allow candidate solutions of varying lengths, and where the length of the optimal solution is thereby a priori unknown. We suggest that non-uniform mutation rates can be beneficial when solving such problems. In particular, we consider a mutation operator that flips each bit with a probability that is inversely proportional to the bit position, rather than the bitstring length. The runtime of the $(1+1)$ EA using this mutation operator is analysed rigorously on standard example functions. Furthermore, the behaviour of the new mutation operator is investigated empirically on a real world software engineering problem that has variable, and unknown solution lengths. The results show how the speedup that can be achieved with the new operator depends on the distribution of the solution lengths in the solution space. We consider a truncated geometric distribution, and show that the new operator can yield exponentially faster runtimes for some parameters of this distribution. The experimental results show that the new mutation operator leads to dramatically shorter runtimes on a class of instances of the software engineering problem that is conjectured to have short solutions on average.
\end{abstract}

\section{Categories and Subject Descriptors}

G.3 [Probability and Statistics]: Probabilistic Algorithms; F.2.2 [Analysis of Algorithms and Problem Complexity]: Nonnumerical Algorithms and Problems-Computations on discrete structures; D.2.5 [Software Engineering]: [Testing tools (e.g., data generators, coverage testing)]

\section{General Terms}

Algorithms, Theory

Permission to make digital or hard copies of all or part of this work for personal or classroom use is granted without fee provided that copies are not made or distributed for profit or commercial advantage and that copies bear this notice and the full citation on the first page. To copy otherwise, to republish, to post on servers or to redistribute to lists, requires prior specific permission and/or a fee.

FOGA'11, January 5-9, 2011, A-6867 Schwarzenberg, Austria.

Copyright 2011 ACM 978-1-4503-0633-1/11/01 ...\$10.00.

\section{Keywords}

runtime analysis, mutation operator, variable-length solutions, search-based software testing, FSM testing, unique input-output sequences

\section{INTRODUCTION}

Effective use of evolutionary algorithms (EAs) often requires the user to find parameter settings for the EA that are appropriate for the problem instance at hand. Experimental studies have suggested that EAs with some kind of adaptation can outperform standard ones. Empirical studies of the advantages of using adaptive parameter values as opposed to fixed values raised an interest in this field $[16,15]$. Among others, such as the selective pressure and crossover rate, one of the most important parameter settings in EAs that use the bitwise mutation operator is the mutation rate. Adapting the mutation rate to the problem specifics will, when carefully chosen, produce better and robust results. The benefits in solving real-world problems would be of having a speedup of the search process, and to free the end user from the task of selecting suitable parameter values using a time-consuming trial and error process [7]. In particular, when the candidate solutions are represented by bitstrings of length $n$, a mutation rate of $1 / n$ seems effective for some problems. Intuitively, this choice seems appropriate, since the operator will flip one bit in expectation, while still having a good probability of flipping more bits.

Theoretical investigations of the $(1+1)$ EA show that a mutation rate of $\Theta(1 / n)$ is asymptotically optimal for this EA on some simple functions. Droste et al [4] proved that if the mutation rate is decreased to $1 / \alpha(n) n$, or increased to $\alpha(n) / n$, for any function $\alpha(n)=\omega(1)$, then the expected runtime of the $(1+1)$ EA increases from $O(n \log n)$ to $\Omega(\alpha(n) n \log n)$ on OnEMAX. Deviating the mutation rate from $1 / n$ by smaller, i. e. constant, factors has a weaker impact on the asymptotic expected runtime. Doerr and Goldberg recently proved that the $(1+1)$ EA with mutation rate $c / n$, for any constant $c>0$, can optimise any linear function in expected time $O(n \log n)$ [2]. It is important to point out that these results do not necessarily hold for more complex fitness functions and EAs. Jansen and Wegener presented functions where the $(1+1)$ EA with a fixed mutation rate $1 / n$ has super-polynomial runtime, whereas the runtime decreases to a polynomial when the mutation rate is increased to $\Theta(\log n / n)$ [8]. Doerr et al [3] showed that the mutation rate can be essential on strictly monotone functions, a class of functions that contains the linear functions. Consid- 
ering the $(1+1)$ EA with mutation rate $c / n$ for a constant $c$, the expected runtime is $\Theta(n \log n)$ when $c<1$. However, for higher mutation rates where $c>33$, the runtime is $2^{\Omega(n)}$ with an overwhelmingly high probability [3]. For non-elitistic, population-based EAs, the mutation rate can be particularly critical, and must in general be set in accordance with the selective pressure in the EA $[12,11]$. Keeping in mind these restrictions, a mutation rate of $1 / n$ is often a simple choice in the absence of problem-specific knowledge.

However, setting the mutation rate to $1 / n$ is only possible when the solution length $n$ is known a priori. While this assumption is implicitly made in most theoretical studies, it does not hold for many practical optimisation problems. These problems often allow candidate solutions of different lengths, and the length of an optimal solution is typically not known. An example of such a problem, which we will discuss in Section 5, is the construction of testing sequences for finite state machines.

The purpose of this paper is to propose and analyse a mutation operator for optimisation problems where the solution lengths are a priori unknown. The paper is organised as follows. Section 2 formalises problems with variablelength solutions within the black-box scenario, and discusses how to quantify the runtime of algorithms working on such problems. Section 3 describes the mutation operator. The theoretical feasibility of the new mutation operator is then justified by runtime analysis on classical example functions in Section 4. Finally, in Section 5, we report experimental results on instances of a software engineering problem. A standard $(1+1)$ EA is used as a baseline in both theoretical and experimental studies. Both strengths and limitations of the proposed approach are discussed in the conclusion.

\section{VARIABLE-LENGTH PROBLEMS}

We now formalise variable solution length problems within the black-box scenario [5]. In the classical black-box scenario, one considers an algorithm $A$ and a class $\mathcal{F}$ of pseudoBoolean functions $\left\{f_{n}:\{0,1\}^{n} \rightarrow \mathbb{R}\right\}$. An adversary selects a function $f$ from this class. The algorithm knows the problem class $\mathcal{F}$, and in particular the solution length $n$, but has no information about the function chosen by the adversary. The goal of the algorithm is to find the optimum of function $f$ by querying function values. The expected runtime of algorithm $A$ on function $f$ is the expected number of times the algorithm queries for a function value, before the optimum is evaluated for the first time. The expected runtime of algorithm $A$ on the class $\mathcal{F}$ is the maximal expected runtime of $A$ on any function in the class.

A variable-length problem consists of a function class $\mathcal{F}_{n}$ and a solution length distribution $K(n)$ over the integers from 1 to $n$. The class of functions is partitioned into subclasses $\mathcal{F}_{n}=\mathcal{G}_{1} \cup \mathcal{G}_{2} \cup \cdots \cup \mathcal{G}_{n}$, where each sub-class $\mathcal{G}_{k}, 1 \leq k \leq n$, contains a set of pseudo-Boolean functions $f:\{0,1\}^{k} \rightarrow \mathbb{R}$, corresponding to solution length $k$.

This time, the solution length $k$ is sampled according to distribution $K(n)$, and $k$ is unknown to the algorithm. Then, the adversary selects one of the functions $f_{k}$ in the corresponding sub-class $\mathcal{G}_{k}$. To not reveal the number of input variables to the algorithm, the function is presented to the algorithm as a padded black-box function $\bar{f}_{k}$ that takes $n$ input variables, defined as

$$
\bar{f}_{k}\left(x_{1} x_{2} \cdots x_{k} x_{k+1} \cdots x_{n}\right):=f_{k}\left(x_{1} x_{2} \cdots x_{k}\right) .
$$

Hence, the function value of $\bar{f}_{k}$ is only determined by the first $k$ bits in $x$. The algorithm knows the problem class $\mathcal{F}$, the maximal solution length $n$, and the solution length distribution $K(n)$. However, the algorithm has no information about the function $f_{k}$ chosen by the adversary, or the sampled solution length $k$. The runtime of the search heuristic $A$ on function $\bar{f}_{k}$ is the number of times search heuristic $A$ evaluates function $\bar{f}_{k}$ until an optimal search point is evaluated for the first time. The expected runtime on the sub-class $\mathcal{G}_{k}$ is the worst case expected runtime of all functions in the class. Finally, the expected runtime on the entire class, denoted $T_{A, \mathcal{F}, K}$ is the expected runtime on $\mathcal{G}_{K}$, where $K$ is sampled according to distribution $K(n)$. In short, this will be referred to as the $K$-length runtime. Notice that this runtime is a random variable, and a function of random variable $K$ specifying the underlying problem dimension. Clearly, the variable length runtime depends on the distribution $K$.

The runtime will be expressed as a function of two variables, the maximal solution length $n$, and a parameter $\varepsilon$ that will control the distribution of $K$, i.e. the solution lengths. We will consider the case where $K$ follows a variant of the geometrical distribution which is truncated to the set of integers $\{1,2, \ldots, n\}$. We call this distribution pseudogeometric. Several of the results obtained in this paper can easily be generalised to other distributions.

Definition 1 (Pseudo-geometric distribution). A random variable $X$ is said to follow a pseudo-geometric distribution with respect to the parameters $n \geq 1$ and $p \in\left(0, \frac{1}{n}\right]$ if

$$
\operatorname{Pr}[X=k]:= \begin{cases}(1-p)^{k-1} p & \text { if } k \in\{1,2, \ldots, n-1\} \\ (1-p)^{n-1} & \text { if } k=n, \text { and } \\ 0 & \text { otherwise. }\end{cases}
$$

For notational convenience, we will use the parameter setting $p:=n^{-\varepsilon}$, where $\varepsilon \in(0,1)$, and consider $\varepsilon$ as the parameter of the pseudo-geometric distribution. It is easy to see that the expectation of a pseudo-geometric random variable is asymptotically the same as the expectation of a geometric random variable.

Proposition 1. If $K$ is a pseudo-geometrically distributed random variable with parameter $p \geq n^{-\varepsilon}$ for $0<\varepsilon<1-\delta$, where $\delta>0$ is an arbitrarily small constant, then $\mathbf{E}[K]=$ $\Theta(1 / p)$.

Proof. For the lower bound, we have

$$
\begin{aligned}
\mathbf{E}[K] & \geq(1 / p) \operatorname{Pr}[K \geq 1 / p] \\
& \geq(1 / p)(1-p)^{1 / p-1} \\
& \geq 1 / e p .
\end{aligned}
$$

For the upper bound, Eq. (2) in the appendix gives

$$
\begin{aligned}
\mathbf{E}[K] & \leq n(1-p)^{n-1}+\frac{p}{1-p} \sum_{k=1}^{\infty} k(1-p)^{k} \\
& =e^{-\Omega\left(n^{\delta}\right)}+\frac{p}{(1-p)} \frac{(1-p)}{p^{2}} \\
& =O(1 / p) .
\end{aligned}
$$




\section{POSITION-DEPENDENT MUTATION}

The $(1+1)$ EA using the new mutation operator can be described as follows.



The only difference with the standard $(1+1)$ EA is that we generalise the mutation rate in step 4 to $\chi(i)$. The classical $(1+1) \mathrm{EA}$ is obtained by setting the mutation rate function to $\chi(i):=1 / n$. In the following, we will compare this algorithm to the variant called $(1+1) \mathrm{EA}_{i}$ where the mutation rate is defined as $\chi(i):=1 /(i+1)$. This algorithm flips early bit positions more often than later bit positions. In particular, bit position 1 is flipped in expectation every second iteration, whereas bit position $n$ is flipped in expectation only once every $n+1$ iterations.

The intuitive argument behind setting $\chi(i)=1 /(i+1)$ is that for any solution length $k$, the majority of the bit positions will have the "right" asymptotic mutation rate of $\Theta(1 / k)$. More precisely, for an arbitrarily small constant $\alpha>0$, all the bit positions between $\alpha k$ and $k$ have mutation rate $\Theta(1 / k)$, where the asymptotic notation is with respect to variable $k$. A counterargument is that there is still a constant fraction of the bit positions where the mutation rate is too high, namely the positions from 1 to $\alpha k$. In particular, the leading bit positions will be flipped very often. In the next section, we prove that the negative effects of having too high mutation rate in the first $\alpha k$ positions is out-weighted by the benefit of having the "right" mutation rate on the last $(1-\alpha) k$ positions.

\section{THEORETICAL RUNTIME ANALYSIS}

The goal of this section is to analyse the runtime of the $(1+1)$ EA with the position-dependant mutation operator on problems with unknown solution lengths. To this aim, we select the example functions ONEMAX and LEADINGONES, which are commonly considered in runtime analysis of new search heuristics. In the next experimental section, we will consider the UIO problem, which is a software testing problem. Note that there exist instances of the UIO problem that correspond exactly to the ONEMAX and LEADINGONES problems.

Before presenting the runtime analysis, we restate Jensen's inequality, which turns out to be a very useful tool in our scenario.

Lemma 1 (JEnsen's InEQuALity [14]). Let $X$ be a random variable with values in the interval $(a, b)$. If $f$ is a convex function over the interval $(a, b)$, then

$$
f(\mathbf{E}[X]) \leq \mathbf{E}[f(x)] .
$$

If $f$ is a concave function over the interval $(a, b)$, then

$$
f(\mathbf{E}[X]) \geq \mathbf{E}[f(x)] .
$$

The direct consequence of Jensen's inequality is that if the expectation of the random variable $T_{A, f, K}$ conditional on the event $K=k$ can be expressed as a convex function $g(k)$, then $g(\mathbf{E}[K])$ is a lower bound on the unconditional expectation of $T_{A, f, K}$. On the other hand if the expectation of $T_{A, f, K}$ conditional on the event $K=k$ can be expressed as a concave function $h(k)$, then $h(\mathbf{E}[K])$ is an upper bound on the unconditional expectation of $T_{A, f, K}$.

\subsection{Simple Pseudo-Boolean Functions}

We will first consider the function

$$
\operatorname{LEADINGONES}(x)=\sum_{i=1}^{n} \prod_{j=1}^{i} x_{j} .
$$

Our goal is to describe how the expected runtime depends on the distribution of the solution lengths, i.e. on the distribution parameter $\varepsilon$.

Theorem 1. If $K$ is a pseudo-geometrically distributed random variable with parameter $p=1 / n^{\varepsilon}$, where $\varepsilon \in(0,1-$ $\delta)$ for any constant $\delta \in(0,1)$, then the expected $K$-length runtime of $(1+1) E A_{i}$ on LEADINGONES $K$ is $\Theta\left(n^{3 \varepsilon}\right)$.

Proof. We start with the lower bound, and condition on the event $K=k$, for some $k, 1 \leq k \leq n$. It simplifies the analysis if we only consider the optimisation process during the period in which the current search point has at least $2 k / 3$ leading 1-bits, and less than $k-2$ leading 1-bits, and do not account for the other time steps. So, let us assume that the current search point has $i, 2 k / 3 \leq i<k-2$, leading 1-bits. If the EA flips none of the first $i$ bits, and flips the 0 -bit in position $i+1$, then a search point with at least $i+1$ leading 1-bits is obtained. However, such a search point may have more than $i+1$ leading 1-bits, depending on the bit values in position $i+2$ and onwards. For the lower bound, we need to account for such 1-bits that the algorithm gains for "free".

More precisely, a bit-position $i+2$ in a given run is called a free-rider if the first accepted search point with at least $i+1$ leading 1-bits has a 1-bit in position $i+2$. Following arguments similar to those in [4], it can be shown that the bits after the first 0-bit in the current search point are uniformly distributed. Hence, the number of free-riding bit positions among the last $k / 3$ bit positions corresponds to the number of 1-bits in a random bitstring of length $k / 3$. By a Chernoff bound, the probability of the event that there are more than $k / 4$ free-riders is $e^{-\Omega(k)}$. We call this event a failure. Hence, with overwhelmingly high probability in $k$, we must wait for the EA to flip the left-most 0-bit in the current search point at least $k / 3-k / 4=k / 12$ times.

In order to increase the number of leading 1-bits, all the first $i \geq 2 k / 3$ bits must remain unchanged. The probability of this event is no more than

$$
\prod_{j=1}^{2 k / 3}\left(1-\frac{1}{j+1}\right)=\prod_{j=1}^{2 k / 3} \frac{j}{j+1}=\frac{1}{2 k / 3+1} .
$$

In addition, bit position $i+1 \geq 2 k / 3+1$ must be flipped. Hence, the probability of increasing the number of leading 1-bits from $i$ is no more than $1 /(2 k / 3+1)(2 k / 3+2)=: q$.

Taking into account that this event must happen at least $k / 12$ times, the expected number of iterations to reach an optimum is therefore at least $(k / 12)(1 / q)=\Omega\left(k^{3}\right)$. The 
function $f(k)=k^{3}$ satisfies $f^{\prime \prime}(k) \geq 0$ on the interval [1, $n$, hence Jensen's inequality can be applied with respect to the expectation of $K$ given in Proposition 1. Also taking into account the failure probability $e^{-\Omega(k)}$, the unconditional $K$ length runtime is $\Omega\left(n^{3 \varepsilon}\right)$.

We now prove the upper bound. Improving a solution from a position with $i$ leading 1-bits is achieved by flipping bit position $i+1$, and flipping none of the first $i$ bit positions. The probability of this event is

$$
q_{i} \geq \frac{1}{i+2} \prod_{j=1}^{i}\left(1-\frac{1}{i+1}\right)=\frac{1}{(i+1)(i+2)} \geq \frac{1}{2 i^{2}}
$$

Hence, the expected time to obtain the optimum is no more than $\sum_{i=1}^{k} 1 / q_{i} \leq 2 k^{3}$, and an upper bound on the unconditional $K$-length runtime is

$$
\begin{aligned}
\mathbf{E}[T] & \leq \sum_{k=1}^{n-1} 2 k^{3} \operatorname{Pr}[K=k] \\
& \leq \frac{2 p}{(1-p)} \sum_{k=1}^{\infty} k^{3}(1-p)^{k} \\
& =\frac{2 p}{(1-p)} \frac{(1-p)\left(1+4(1-p)+(1-p)^{2}\right)}{p^{4}} \\
& =O\left(p^{-3}\right) \\
& =O\left(n^{3 \varepsilon}\right),
\end{aligned}
$$

where the third step follows from Eq. (4) in the appendix.

We then consider the (1+1) EA with the classical mutation operator. It is well known that this algorithm has expected optimisation time $\Theta\left(n^{2}\right)$ on the LEADINGONES problem with fixed solution length [4]. While the variable-length optimisation time reduces with shorter expected solution lengths, the following theorem shows that the expected optimisation time of the $(1+1)$ EA is never sub-linear on this problem.

Theorem 2. If $K$ is a pseudo-geometrically distributed random variable with $p=1 / n^{\varepsilon}$, where $\varepsilon \in(0,1-\delta)$ for any constant $\delta \in(0,1)$, then the expected $K$-length runtime of the $(1+1) E A$ on $\operatorname{LEADINGONES}_{K}$ is $\Theta\left(n^{1+\varepsilon}\right)$.

Proof. We condition on the event $K=k$. Given a current search point with $i, 0 \leq i<k$, leading 1-bits, the probability of making an improving step is at least (1$1 / n)^{k}(1 / n) \geq 1 /$ en and at most $1 / n$. The EA must make at most $k$ improving steps, and by the same arguments as in the proof of Theorem 1, at least $k / 12$ improving steps. Hence, the expected runtime is at least $n k / 12$ and at most enk. Note that the function $f(k)=c n k$ is both convex and concave for any constant $c$. Jensen's inequality with respect to the expectation of $K$ given in Proposition 1 therefore implies that the expected $K$-length runtime is $\Theta(n / p)=$ $\Theta\left(n^{1+\varepsilon}\right)$.

We are now interested in determining when the $(1+1) \mathrm{EA}_{i}$ outperforms the standard $(1+1) \mathrm{EA}$, and vice versa. For this purpose, we define the expected speedup as the ratio between the expected $K$-length runtimes of the algorithms.

Corollary 1. If $K$ is a pseudo-geometrically distributed random variable with $p=1 / n^{\varepsilon}$, where $\varepsilon \in(0,1-\delta)$ for any constant $\delta \in(0,1)$, then the expected speedup of $(1+1) E A_{i}$ over $(1+1)$ EA on LEADINGONES $_{K}$ is $\Theta\left(n^{1-2 \varepsilon}\right)$.
This result means that the $(1+1)$ EA outperforms the $(1+1) \mathrm{EA}_{i}$ when $\varepsilon>1 / 2$, i.e. when the average solution length is above $\sqrt{n}$. In the worst case, when the solution length is fixed to $n$, the $(1+1) \mathrm{EA}_{i}$ is a factor of $n$ slower than the $(1+1)$ EA. On the other hand, $(1+1) \mathrm{EA}_{i}$ outperforms the $(1+1)$ EA when $\varepsilon<1 / 2$, i.e. when the average solution length is below $\sqrt{n}$. In particular, for $\varepsilon=\log \log n / \log n$, the $(1+1) \mathrm{EA}_{i}$ is exponentially faster than the $(1+1) \mathrm{EA}$ ! In this case, the $(1+1) \mathrm{EA}_{i}$ has expected runtime $O(\log n)$, whereas the $(1+1)$ EA has expected runtime $\Omega(n)$. (Recall that the expected waiting time until $(1+1)$ EA flips a specific bit is $n$.)

We now consider another well known fitness function,

$$
\operatorname{OnEMax}(x):=\sum_{i=1}^{n} x_{i} \text {. }
$$

Theorem 3. The expected running time of the $(1+1) E A_{i}$ on $\operatorname{ONEMAX}_{K}$ whose shortest optimal solution is of length $K$ is $O\left(n^{2 \varepsilon} \log n\right), K$ being a pseudo-geometrically distributed random variable with parameter $p=n^{-\varepsilon}$, for a constant $\varepsilon, 0<\varepsilon<1$.

Proof. We condition on the event $K=k$. Given that the current search point $x$ has $i, 0 \leq i<k$, 0 -bits, the probability of increasing the number of 1 -bits is

$$
\begin{aligned}
p_{i} & \geq \sum_{\substack{j=1 \\
x_{j}=0}}^{k} \frac{1}{j+1} \prod_{\substack{r=1 \\
r \neq j}}^{k}\left(1-\frac{1}{r+1}\right) \\
& \geq \frac{i}{k+1} \prod_{r=1}^{k} \frac{r}{r+1} \\
& =\frac{i}{(k+1)^{2}} .
\end{aligned}
$$

Hence, the expected time to obtain $k$ 1-bits is no more than $\sum_{i=1}^{k-1} 1 / p_{i}=O\left(k^{2} \log k\right)$. The expected $K$-length runtime is thus

$$
\begin{aligned}
\mathbf{E}[T] & =\sum_{k=1}^{n} \mathbf{E}[T \mid K=k] \mathbf{P r}[K=k] \\
& =O\left(n^{2} \log n\right)(1-p)^{n-1}+\sum_{k=1}^{n} O\left(k^{2} \log k\right) p(1-p)^{k-1} \\
& \leq O(\log n) \frac{p}{(1-p)} \sum_{k=1}^{\infty} k^{2}(1-p)^{k}=O\left(n^{2 \varepsilon} \log n\right),
\end{aligned}
$$

where the last step follows from Eq. (3) in the appendix.

To prove a lower bound for $(1+1) \mathrm{EA}_{i}$ on $\mathrm{ONEMAX}_{K}$, it is necessary to take into account the positions of the 0-bits in the bitstring. In the most favourable case for the algorithm, all the 0-bits are located in the beginning of the bitstring, and the algorithm can easily flip many 0-bits. However, we think the 0-bits are unlikely to be positioned in such a way. We conjecture that the bound in Theorem 3 is tight when the distribution parameter $\varepsilon$ is constant. On the other hand, for the classical $(1+1) \mathrm{EA}$, we are able to prove a tight bound on the expected runtime.

Theorem 4. Let $K$ be pseudo-geometrically distributed with parameter $p=1 / n^{\varepsilon}$ where $\epsilon \in(0,1)$. Then the expected $K$-length running time of the $(1+1)$ EA on $\mathrm{ONEMAX}_{K}$ is $\Theta(\varepsilon n \log n)$. 
Proof. For the lower bound, we first calculate the expected runtime conditional on a fixed solution length $k$. By Chernoff bounds, the probability that there are more than $2 k / 3$ 1-bits in the initial search point is bounded from above by $e^{-\Omega(k)}$. The probability that one of the remaining $k / 3$ 0 -bits has not been mutated within $n \ln k$ steps is

$$
\begin{aligned}
1-\left(1-\left(1-\frac{1}{n}\right)^{n \ln k}\right)^{k / 3} & \geq 1-\left(1-\frac{1}{k}\right)^{k / 3} \\
& =\Omega(1) .
\end{aligned}
$$

Therefore we have $\mathbf{E}[T \mid K=k]=\Omega(n \log k)$. In particular, the expected runtime for $K=n^{\varepsilon}-1$, i. e. slightly below the expectation of $K$ is $\Omega(\varepsilon n \log n)$. The probability that $K$ takes at least value $n^{\varepsilon}-1$ is

$$
\operatorname{Pr}\left[K \geq n^{\varepsilon}-1\right] \geq(1-p)^{n^{\varepsilon}-1} \geq 1 / e .
$$

So a lower bound on the unconditional expected $K$-length runtime is

$$
\mathbf{E}\left[T \mid K \geq n^{\varepsilon}-1\right] \operatorname{Pr}\left[K \geq n^{\varepsilon}-1\right]=\Omega(\varepsilon n \log n) .
$$

Following the same ideas as in the proof of Theorem 3, one can show that the expected runtime conditional on the event $K=k$ is $O(n \log k)$. An upper bound of $O(\varepsilon n \log n)$ now follows from Jensen's inequality.

It is worth noting that assuming the parameter $\varepsilon$ is constant, the $(1+1)$ EA has asymptotically no advantage of shorter solution lengths. The speedup of $(1+1) \mathrm{EA}_{i}$ over $(1+1)$ EA on $\operatorname{ONEMAX}_{K}$ is similar to that on LEADINGONES ${ }_{K}$. We did not prove any lower bound on the runtime of $(1+1) \mathrm{EA}$ on $\mathrm{ONEMAX}_{K}$, hence we can only show a lower bound on the speedup for this fitness function.

Corollary 2. If $K$ is a pseudo-geometrically distributed random variable with $p=n^{-\varepsilon}$, for a constant $\varepsilon, 0<\varepsilon<1$, then the expected speedup of $(1+1) E A_{i}$ over $(1+1) E A$ on $\mathrm{ONEMAX}_{K}$ is $\Omega\left(n^{1-2 \varepsilon}\right)$.

\section{EXPERIMENTAL RUNTIME ANALYSIS}

The previous section provided rigorous results on the expected runtime of the two algorithms on example problems having a clear and simple structure. In practical optimisation settings, the problem structure can often be less clear, or unknown. In this section, we will experimentally compare the performance of the two algorithms on the problem of constructing so-called unique input-output sequences, which is a problem with less clear structure. See [13] for a discussion of how experimental investigations can complement a theoretical runtime analysis.

\subsection{Problem Definition}

Definition 2 (Finite State Machine [10]). A finite state machine (FSM) $M$ is a quintuple $M=(I, O, S, \delta, \lambda)$, where $I$ is the set of input symbols, $O$ is the set of output symbols, $S$ is the set of states, $\delta: S \times I \rightarrow S$ is the state transition function and $\lambda: S \times I \rightarrow O$ is the output function.

For notational convenience, we extend the domain of the state transition function $\delta$ and the output function $\lambda$ to the set of non-empty strings over the input alphabet, i.e.

$$
\begin{aligned}
& \delta\left(s, a_{1} a_{2} \cdots a_{n}\right):=\delta\left(\delta\left(s, a_{1} a_{2} \cdots a_{n-1}\right), a_{n}\right), \text { and } \\
& \lambda\left(s, a_{1} a_{2} \cdots a_{n}\right):=\lambda\left(s, a_{1}\right) \cdot \lambda\left(\delta\left(s, a_{1}\right), a_{2} \cdots a_{n}\right) .
\end{aligned}
$$

Definition 3 (Unique InPut-Output Sequence [9]). $A$ unique input-output sequence (UIO) for a state $s$ in an FSM $M$ is a string $x$ over the input alphabet of $M$ such that $\lambda(s, x) \neq \lambda(t, x)$ for all states $t, t \neq s$.

The interesting aspect of the UIO problem in the context of this paper is that the length of the UIOs are a priori unknown, and depends greatly on the problem instance. The shortest UIO sequence can be exponentially long with respect to the number of states [9]. On the other hand, experiments show that UIO sequences are typically short [1]. Trakhtenbrot and Barzdin considered a random FSM model with $n$ states and $m$ output symbols, showing that with probability $1-O(1 / n)$, any pair of distinguishable states in a random FSM can be distinguished by a sequence of no longer than $c \ln n / \ln m$, for some constant $c$ [17].

To construct UIOs using an evolutionary algorithm, we need to find a way of representing and evaluating the quality of candidate solutions. Several approaches have been proposed in the literature. Here, we will use the approach described in [13]. Candidate solutions are represented as strings of length $n$ over the input alphabet $I$ of the FSM. The length parameter $n$ specifies the maximal length of sequences among which we want to search for a UIO. We treat the problems of generating UIOs for the various states in the FSM separately, and define one fitness function for each state. UIOs for all the states in the FSM can be obtained simply by re-running the EA, once for each of the fitness functions. In the experiments, we therefore only considered finding UIOs for a designated state $s_{1}$.

Definition 4. Given an FSM M, define the fitness function $f_{M, s}: I^{n} \rightarrow \mathbb{R}$ for state $s$ as

$$
f_{M, s}(x):=|\{t \in S \mid \lambda(s, x) \neq \lambda(t, x)\}| .
$$

Note that if $x$ is an optimal solution, then so is the concatenation $x y$, for any input sequence $y$. Therefore, in analogy with the padding function in Eq. (1), one can consider the fitness function $f_{M, s}$ as a padded version of the true fitness function $f_{k}$, corresponding to a solution of length $k$.

Before describing the details of our experiments, we note that the problem of constructing UIOs has been studied extensively within the field search-based software engineering [6], which intersects evolutionary computation and software engineering. All previous studies used EAs with fixed mutation-rates.

\subsection{Experimental Setup}

Experiments were conducted on two FSM models, called the Random FSM model, and the Counter FSM model. The details of these models will be described in the next subsections. The experiments consisted of a series of runs. A run consists of first generating an instance with $n=100$ states, where the corresponding fitness function was defined with a maximal solution length $n$. The $(1+1) \mathrm{EA}$ and the $(1+1) \mathrm{EA}_{i}$ were then started on this instance. An algorithm was stopped after 20000 iterations, or when an optimal solution had been found. If the algorithm did not find a solution within 20000 iterations, then the run was deemed unsuccessful for the algorithm. 10000 runs were made for the Random FSM model, and 10000 runs were made for the Counter FSM model. The number of iterations used by each algorithm and their success rates were recorded. The number of iterations 
used by each algorithm on the instances was plotted using box-and-whisker plots, indicating the smallest observed iteration number, the lower quartile, the median, the upper quartile, and the largest observed iteration number. The $y$-scale for the Random FSM model was log-transformed.

\subsection{A Random FSM Model}

For the purpose of this paper, we define a random FSM model $R=\left(I, O, S, \delta_{R}, \lambda_{R}\right)$. It has $n$ states $S:=\left\{s_{1}, \ldots s_{n}\right\}$, and binary input and output alphabets $I=O=\{0,1\}$. A random machine is produced by first selecting uniformly at random a permutation $\sigma$ over the states $S$ and a random vector $v$ of $n$ states $v \in S^{n}$. Furthermore, a random bitstring $x$ of length $n$ is sampled, where each bit is 0 with probability $1-10 / n$, and 1 with probability $10 / n$. The output function $\lambda_{R}$ and transition function $\delta_{R}$ are then defined as

$$
\begin{array}{ll}
\delta_{R}\left(s_{i}, 0\right):=\sigma\left(s_{i}\right) & \lambda_{R}\left(s_{i}, 0\right):=0 \\
\delta_{R}\left(s_{i}, 1\right):=v_{i} & \lambda_{R}\left(s_{i}, 1\right):=x_{i} .
\end{array}
$$

The permutation $\sigma$ guarantees that all states in the machine are reachable; Starting in state $s_{1}$, the machine cycles through all the states on input sequence $0^{n}$. The bias towards 0-bits in the output on 1-input, ensures that many states will have the same output on this input, and is intended to make the problem instance harder.

\subsection{A Counter FSM Model}

To contrast the unstructured random FSM, we will also consider the highly structured counter FSM model $C=$ $\left(I, O, S, \delta_{C}, \lambda_{C}\right)$ [13]. The counting machine has $n$ states $S:=\left\{s_{1}, s_{2}, \ldots, s_{n}\right\}$ and binary input and output alphabets $I=O=\{0,1\}$. For all states $s_{i} \in S$, the output function $\lambda_{C}$ is defined as

$$
\lambda_{C}\left(s_{i}, 0\right):=0, \text { and, } \lambda_{C}\left(s_{i}, 1\right):= \begin{cases}1 & \text { if } i=n, \text { and } \\ 0 & \text { otherwise }\end{cases}
$$

and the transition function $\delta_{C}$ is defined as

$$
\delta_{C}\left(s_{i}, 0\right):=s_{i}, \text { and, } \delta_{C}\left(s_{i}, 1\right):= \begin{cases}s_{1} & \text { if } i=n, \text { and } \\ s_{i+1} & \text { otherwise. }\end{cases}
$$

The counter FSM counts the number of 1-symbols in the input, and outputs a 1-symbol if and only if it has received $n$ such inputs. In all other cases, the machine outputs a 0 -symbol. It is known that the shortest UIO for state $s_{1}$ in the counter machine has length $n-1$ [13], and is of the form $1^{n-1}$.

\subsection{Results and Discussion}

The experimental results are summarised in Table 1 and Figure 2. Both algorithms had high success rates on both FSM models. It is difficult to compare the performance of the algorithms based on their success rates. However, the box-and-whisker plots in Figure 2 show that the median runtime of the $(1+1) \mathrm{EA}_{i}$ on the Random FSM model is an order of magnitude lower than the median runtime of the $(1+1)$ EA. Note that the scale is logarithmic. The upper quartile of the runs of $(1+1) \mathrm{EA}_{i}$ had lower runtime than the median runtime of the $(1+1)$ EA on the Random FSM model. The results are different for the Counter FSM model. Here, the median runtime of $(1+1) \mathrm{EA}_{i}$ is higher than the upper quartile runtime of the $(1+1) \mathrm{EA}$.
The results for the Counter FSM is consistent with the theoretical results. The $(1+1)$ EA outperforms the $(1+1) \mathrm{EA}_{i}$ when the solution length is close to what has been selected as the maximal candidate solution length. Random FSM instances are known to have short UIOs, i. e. solutions, on average [17]. The experimental results show that the $(1+1) \mathrm{EA}_{i}$ significantly outperforms the $(1+1)$ EA for such instances.

\section{CONCLUSION}

We have considered optimisation problems where the solution length is a priori unknown. For such problems, we have proposed a parameter-less mutation operator where the mutation rate is non-uniform. The operator can be applied in most evolutionary algorithms, and for many problems that have variable, or unknown solution lengths.

The approach is evaluated theoretically and experimentally using the $(1+1)$ EAs on three different problems, including one real-world FSM testing problem. A rigorous runtime analysis on two example functions shows that the $(1+1) \mathrm{EA}_{i}$ with position-dependant mutation operator can be exponentially faster than the standard $(1+1)$ EA when the solution lengths are short on average. The expected waiting time until the $(1+1) \mathrm{EA}_{i}$ flips a given bit position $k$ is $k+1$, while it is $n$ for the $(1+1)$ EA. Hence, if only the first few bit positions need to be flipped, then the $(1+1) \mathrm{EA}_{i}$ may do so within shorter time than the $(1+1) \mathrm{EA}_{i}$. However, when the solution lengths are long, the modified mutation operator can incur a linear overhead in the expected runtime. Experimental results on computing testing sequences for FSMs are consistent with the theoretical results, showing that the new mutation operator can reduce the runtime significantly on instances that are conjectured to have short solution lengths on average.

A fundamental assumption behind this work is that the value of the cost function only depends on the $k$ left-most variables in the problem, where $k$ is unknown to the algorithm. If this assumption does not hold, e.g. the variable positions are permuted, then the new mutation operator cannot in general be expected to be more efficient than the standard mutation. While this assumption may seem strong, we think it is valid for many practical optimisation problems. For example, the assumption is valid in the practical problem that motivated this work, i. e., the construction of testing sequences for finite state machines.

As future work, we suggest a more extensive runtime analysis, and investigation of the approach in other problem domains where the solution lengths are unknown, or variable.

\section{Acknowledgements}

The main part of this work was done while Stephan Cathabard was a visiting student in The School of Computer Science at The University of Birmingham, UK. This work was supported by Deutsche Forschungsgemeinschaft (DFG) under grant no. WI 3552/1-1, and by EPSRC under grant no. $\mathrm{EP} / \mathrm{D} 052785 / 1$. 


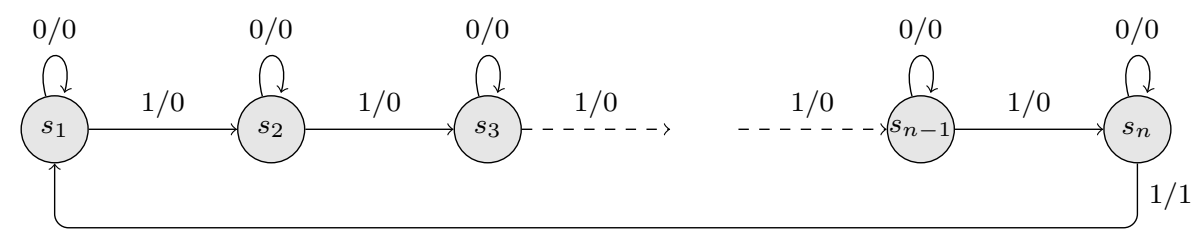

Figure 1: Counter FSM Model.

\begin{tabular}{lll}
\hline Algorithm & Random FSM Model & Counter FSM Model \\
\hline$(1+1)$ EA & $9847 / 10000(98.47 \%)$ & $10000 / 10000(100 \%)$ \\
$(1+1)$ EA $_{i}$ & $9991 / 10000(99.91 \%)$ & $10000 / 10000(100 \%)$ \\
\hline
\end{tabular}

Table 1: Success rates of runs of duration 20000 iterations.
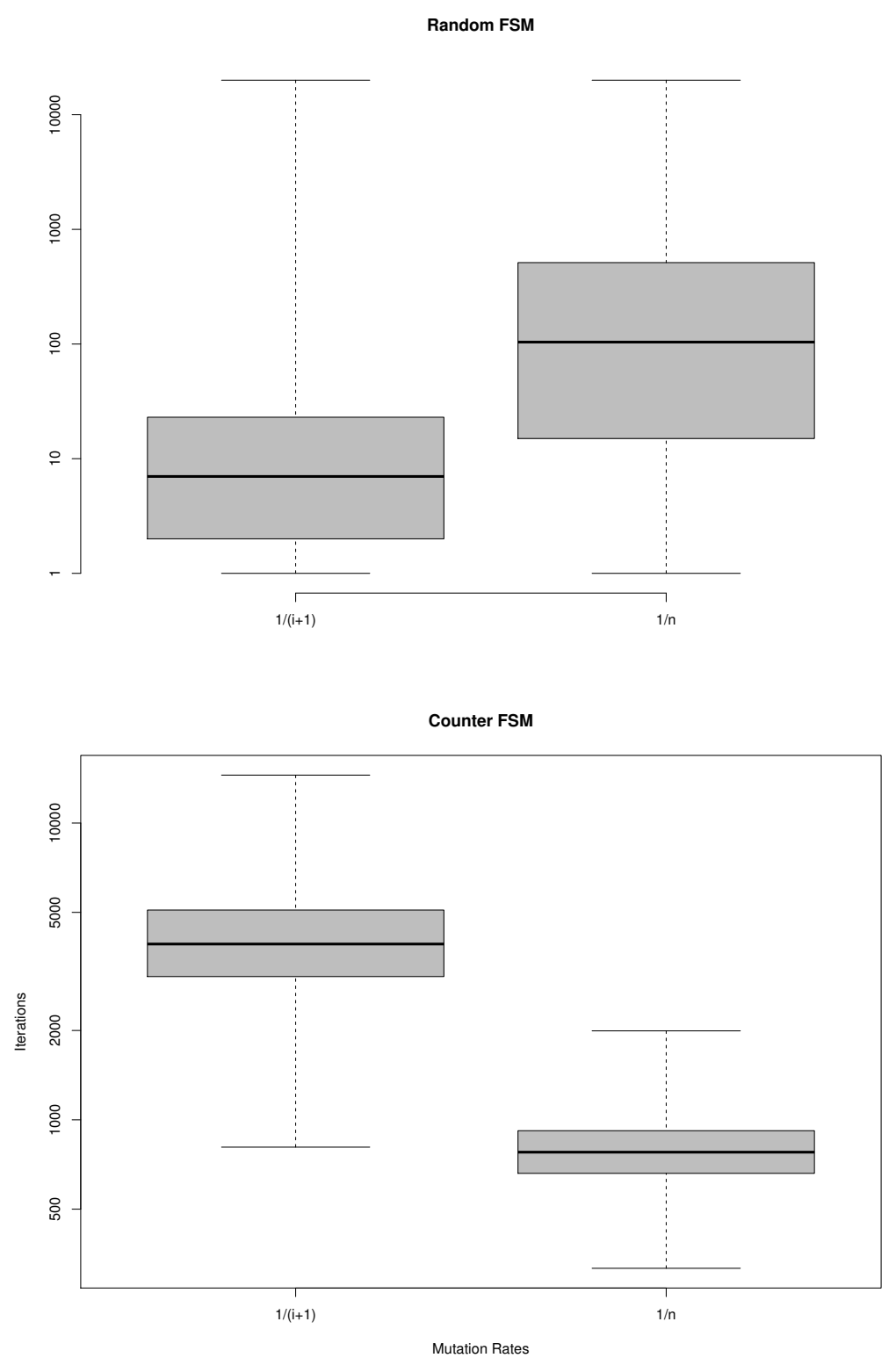

Figure 2: Results of experiments with position-dependant mutation (labelled $1 /(i+1)$, left) and normal mutation rates (labelled $1 / n$, right). 


\section{REFERENCES}

[1] Karnig Agop Derderian, Robert Mark Hierons, Mark Harman, and Qiang Guo. Automated unique input output sequence generation for conformance testing of FSMs. The Computer Journal, 49(3):331-344, 2006.

[2] Benjamin Doerr and Leslie Ann Goldberg. Adaptive drift analysis. In Proceedings of Parallel Problem Solving from Nature - (PPSN XI), pages 32-41, 2010.

[3] Benjamin Doerr, Thomas Jansen, Dirk Sudholt, Carola Winzen, and Christine Zarges. Optimizing monotone functions can be difficult. In Proceedings of the 11th international conference on Parallel problem solving from nature: Part I, PPSN'10, pages 42-51, Berlin, Heidelberg, 2010. Springer-Verlag.

[4] Stefan Droste, Thomas Jansen, and Ingo Wegener. On the analysis of the $(1+1)$ Evolutionary Algorithm. Theoretical Computer Science, 276:51-81, 2002.

[5] Stefan Droste, Thomas Jansen, and Ingo Wegener. Upper and lower bounds for randomized search heuristics in black-box optimization. Theory of Computing Systems, 39(4):525-544, 2006.

[6] Mark Harman, S. Afshin Mansouri, and Yuanyuan Zhang. Search based software engineering: A comprehensive analysis and review of trends techniques and applications. Technical Report TR-09-03, Department of Computer Science, King's College London, 2009.

[7] R. Hinterding, Z. Michalewicz, and A.E. Eiben. Adaptation in evolutionary computation: a survey. In Evolutionary Computation, 199\%., IEEE International Conference on, pages 65 -69, April 1997.

[8] Thomas Jansen and Ingo Wegener. On the choice of the mutation probability for the $(1+1)$ EA. In PPSN VI: Proceedings of the 6th International Conference on Parallel Problem Solving from Nature, pages 89-98, London, UK, 2000. Springer-Verlag.

[9] David Lee and Mihalis Yannakakis. Testing finite-state machines: state identification and verification. IEEE Transactions on Computers, 43(3):306-320, 1994.

[10] David Lee and Mihalis Yannakakis. Principles and methods of testing finite state machines-a survey. Proceedings of the IEEE, 84(8):1090-1123, 1996.

[11] Per Kristian Lehre. Negative drift in populations. In Proceedings of Parallel Problem Solving from Nature (PPSN XI), volume 6238 of LNCS, pages 244-253. Springer Berlin / Heidelberg, 2011.

[12] Per Kristian Lehre and Xin Yao. On the impact of the mutation-selection balance on the runtime of evolutionary algorithms. In FOGA '09: Proceedings of the tenth ACM SIGEVO workshop on Foundations of genetic algorithms, pages 47-58, New York, NY, USA, 2009. ACM.

[13] Per Kristian Lehre and Xin Yao. Runtime analysis of the $(1+1)$ EA on computing unique input output sequences. Information Sciences, In Press., 2010.

[14] Michael Mitzenmacher and Eli Upfal. Probability and Computing: Randomized Algorithms and Probabilistic Analysis. Cambridge University Press, New York, NY, USA, 2005.

[15] Jim Smith and T.C. Fogarty. Self adaptation of mutation rates in a steady state genetic algorithm, 1996.
[16] Dirk Thierens. Adaptive mutation rate control schemes in genetic algorithms. In Proceedings of the 2002 IEEE World Congress on Computational Intelligence: Congress on Evolutionary Computation, pages 980-985. IEEE Press, 2002.

[17] B A Trakhtenbrot. Finite automata; behavior and synthesis. American Elsevier, 1973.

\section{APPENDIX}

For all $x$, where $|x|<1$, it holds that

$$
\begin{aligned}
\sum_{i=1}^{\infty} i x^{i} & =\frac{x}{(1-x)^{2}}, \\
\sum_{i=1}^{\infty} i^{2} x^{i} & =\frac{x(1+x)}{(1-x)^{3}}, \quad \text { and } \\
\sum_{i=1}^{\infty} i^{3} x^{i} & =\frac{x\left(1+4 x+x^{2}\right)}{(1-x)^{4}} .
\end{aligned}
$$

